\title{
A Computational Approach for the Discovery of
}

\section{Protein-RNA Networks}

Domenica Marchese ${ }^{1,2}$, Carmen Maria Livi ${ }^{1,2}$ and Gian Gaetano Tartaglia ${ }^{1,2,3}$

${ }^{1}$ Centre for Genomic Regulation (CRG), Dr Aiguader 88, 08003 Barcelona, Spain

${ }^{2}$ Universitat Pompeu Fabra (UPF), 08003 Barcelona, Spain

${ }^{3}$ Institució Catalana de Recerca i Estudis Avançats (ICREA), 23 Passeig Lluís Companys, 08010 Barcelona, Spain

\section{Summary}

Protein-RNA interactions play important roles in a wide variety of cellular processes, ranging from transcriptional and post-transcriptional regulation of genes to host defense against pathogens. In this chapter we present the computational approach catRAPID to predict protein-RNA interactions and discuss how it could be used to find trends in ribonucleoprotein networks. We envisage that the combination of computational and experimental approaches will be crucial to unravel the role of coding and non-coding RNAs in protein networks

\subsection{Introduction}

The human genome harbors $>1500$ genes encoding proteins annotated to contain at least one RNA-binding domain (RBD) (1). The number of proteins with identified RNA-binding ability (RBP), either possessing canonical or non-canonical RBDs (2), is increasing. The fact that some proteins bind to transcripts through domains or regions that are not specifically evolved to this precise purpose $(3,4)$ is particularly intriguing. Indeed, recent manuscripts suggests a scenario 
where unexpected players can exert crucial functions in domains that were previously thought of as exclusively regulated by selected RBD-containing proteins (5).

Computational models represent an important source of information that can be exploited to identify hidden trends and understand the basics of molecular recognition. As a matter of fact, bioinformatics tools can perform exhaustive analyses and extract distinctive features, hence facilitating the design of new experiments. For example, it has been shown in several studies that the composition of primary protein structure, and the physico-chemical properties associated with it, can be used to describe the amino acid regions that are more likely to be involved in binding to RNA molecules $(6,7)$. Due to the limitations of current experimental approaches, it remains difficult to simultaneously investigate the plethora of RBPs bound to a single transcript and RNA regions that are likely to be involved in the binding. This has resulted in experimentalists having to rely on protein analysis to investigate specific signatures.

We developed an algorithm, catRAPID, to investigate protein-RNA associations involved in regulatory mechanisms (8). We trained catRAPID on a large set of protein-RNA pairs available in the Protein Data Bank to discriminate interacting and non-interacting molecules using the information contained in primary structures. catRAPID relies on the ViennaRNA package (9), which has an accuracy of $\sim 76 \%$ (10), to generate predictions of secondary structure ensembles. These structures are then analyzed to extract information on the pairing profile of each nucleotide. By means of this procedure, the probability of catRAPID predicting a protein-RNA interaction has a $72 \%$ correlation with secondary structure information. However, a higher correlation factor is consistently expected with the enhancement of secondary structure prediction accuracies. As the predictive power of global RNA structure becomes less accurate as the length of the RNA increases (11), we developed the catRAPID fragments module that exploits the RNALfold algorithm (10) to determine interactions for the most stable local structure.

\subsection{Materials}

The catRAPID approach (http://s.tartaglialab.com/page/catrapid_group) $(12,13)$ has been 
developed to predict protein associations with coding and non-coding RNAs $(14,15)($ Table 1$)$. In our method, the contributions of secondary structure, hydrogen bonding and van der Waals' are combined together into the interaction profile:

$\vec{\Phi}_{x}=\alpha_{H} \vec{H}_{x}+\alpha_{W} \vec{W}_{x}+\alpha_{S} \vec{S}_{x}$

where the variable $x$ indicates RNA $(x=r)$ or protein $(x=p)$. The hydrogen bonding profile, denoted by $\vec{H}$, is the hydrogen bonding ability of each amino acid (or nucleotide) in a protein (or RNA) sequence:

$\vec{H}=H_{1}, H_{2}, \ldots, H_{\text {length }}$

Similarly, $\vec{S}$ represents the secondary structure occupancy profile and $\vec{W}$ the van der Waals' profile. The interaction propensity $\pi$ is defined as the inner product between the protein propensity profile $\vec{\Psi}_{p}$ and the RNA propensity profile $\vec{\Psi}_{r}$ weighted by the interaction matrix I:

$\pi=\vec{\Psi}_{p} I \vec{\Psi}_{r}$

The algorithms to compute protein-RNA interactions are available at our group webpage http://service.tartaglialab.com/page/catrapid_group:

2.1 catRAPID graphic. Our original algorithm predicts the interaction propensity of a protein-RNA pair reporting the discriminative power DP, which is a measure of interaction strength with respect to the training sets (12). DP ranges from $0 \%$ (the case of interest is predicted to be negative) to $100 \%$ (the case of interest is a positive). DP values above $50 \%$ indicate that the interaction is likely to take place, whereas DPs above $75 \%$ represent high-confidence predictions. Due to computational requirements (intense CPU usage), the catRAPID graphic algorithm accepts protein sequences with a length ranging between 50aa and 750aa and RNA sequences between 50nt and 1200nt (15). 
2.2 catRAPID fragments. When input sequences exceed the length compatible with our computational requirements (i.e.: protein length $>750$ aa or RNA length $>1200 n t$ ), the catRAPID graphic cannot be used to calculate the interaction propensity $(14,15)$. To overcome this limitation, we developed a procedure called fragmentation, which involves division of polypeptide and nucleotide sequences into fragments followed by prediction of the interaction propensities. Two types of fragmentation are possible:

- $\quad$ Protein and RNA uniform fragmentation (transcripts < 10000nt) (15): The fragmentation approach is based on the division of protein and RNA sequences into 104 overlapping segments. This analysis of fragments is particularly useful to identify regions involved in the binding 7,63 .

- $\quad$ Long RNA weighted fragmentation (for transcripts > $10000 \mathrm{nt}$ ) (14): The use of RNA fragments is introduced to identify RNA regions involved in protein binding. The RNALfold algorithm from the Vienna package is employed to select RNA fragments in the range 100200 nt with predicted stable secondary structure).

2.3 catRAPID strength. We previously observed that the strength correlate with chemical affinities (14), which suggests that the interactions propensity can be used to estimate the strength of association (16). This algorithm calculates the strength of a protein-RNA pair with respect to a reference set (13). Random associations between polypeptide and nucleotide sequences are used to build the reference set. Since little interaction propensities are expected from random associations, the reference set is considered a negative control. Reference sequences have the same lengths as the pair of interest to guarantee that the interaction strength is independent of protein and RNA lengths. The interaction strength ranges from 0\% (non-interacting) to $100 \%$ (interacting). Interaction strengths above $50 \%$ indicate propensity to bind.

\section{1 catRAPID applications: self-regulatory mechanisms control protein production}

We used the catRAPID method to unravel self-regulatory pathways (autogenous interactions) controlling gene expression (15). We discovered that aggregation-prone and structurally 
disordered proteins have a strong propensity to interact with their own mRNA (17). Our results (15, 18) are in agreement with previous experimental work:

- It has been shown that the amyloidogenic TAR DNA binding protein 43 TDP-43 and Fragile $X$ mental retardation protein FMRP interact with the 3' UTR of their own mRNA to control protein production $(15,19,20)$. As overexpression leads to high protein concentration and enhanced amyloidogenicity $(21,22)$, it is possible that autogenous interactions prevent from generation of potentially toxic aggregates.

- $\quad$ The biosynthesis of tumor suppressor p53 is controlled by a translational autoregulatory feedback mechanism where the p53 protein binds to its own mRNA in the 5' terminal region, resulting in translational repression (23). Indeed, it has been reported that naturally occurring mutations of p53 are associated with an increase of the aggregation potential (24). In these regards, self-regulation of p53 can be regarded as a way to control its aggregation potential.

- HSP70, the major stress-induced heat shock protein, regulates its own expression by interacting with its mRNA. Prolonged presence of HSP70 is detrimental for the cell, as it promotes aggregation. From ex vivo experiments, it has been shown that an increase in the degradation of HSP70 mRNA accompanies aggregation of HSP70 (25). The interaction of HSP70 with its own mRNA (3' UTR) suggests a self-limiting mechanism to reduce chaperone production and avoid potential toxic effects in absence of stress (25).

- Moreover, the content of ribosomal proteins in eukaryotic cells is controlled by changes in the degradation rate of newly synthesized proteins. Such a high degree of coordination is achieved trough the use of common regulatory elements in the genes and mRNAs of ribosomal proteins. In the majority of cases, regulation follows a feedback pattern, involving interactions of a ribosomal protein with its own pre-mRNA. This regulatory mechanism provides the required level of each individual ribosomal protein in the cell independently of other ribosomal proteins, which is crucial for extra-ribosomal functions. In the case of ribosomal proteins rpS26 and rpS13, high affinity for pre-mRNA fragments containing first introns has been found (26). 


\section{2 catRAPID applications: X-chromosome dosage compensation}

Dosage compensation of sex chromosomes equalizes expression of X-linked genes in organisms where males and females have a different number of $X$ chromosomes. In mammals, Xistmediated $\mathrm{X}$ chromosome inactivation $(\mathrm{XCl})$ implies a complex network of macromolecular associations orchestrated by epigenetic modifiers as well as splicing and transcription factors.

- We used catRAPID to investigate the interactions of the long non-coding Xist with SUZ12, EZH2, YY1, SAF-A, ASF and SATB1 proteins. In striking agreement with experimental evidence, we predicted protein binding sites and their affinities for Xist regions and used our analysis to integrate the existing model of $\mathrm{XCl}$ into a new framework in which the transcriptional repressor $Y Y 1$ tethers Xist to the $X$ chromosome and nuclear matrix proteins SAF-A and SATB1 guide its translocation (14).

In Drosophila melanogaster, translational inhibition of male-specific msl-2 messenger RNA by female-specific protein SXL is crucial for X-chromosome dosage compensation. Experimental studies identified an RNA-binding protein, UNR, as a fundamental co-repressor recruited by SXL to the 3' UTR of msl-2 mRNA for translation inhibition in females.

- $\quad$ RNA affinity chromatography and UV crosslinking assays show that UNR transcript and its 5' UTR (nucleotides 1-261) efficiently bind to UNR protein, whereas 3' UTR (nucleotides 261-447) does not (27). Our calculations, carried out with catRAPID fragments ("Protein and RNA uniform fragmentation" option), reproduce experimental results in great detail, identifying the cold shock domains (CSD; Figure 1a) (28) involved in autogenous interaction. In agreement with previous reports (27), UNR protein is predicted to bind to purine repeats, such as the guanine-rich region of its 5' UTR (nucleotides 2677; Figure 1b)

\section{3 catRAPID applications: Alternative splicing}

Recent studies indicate that nonsense-mediated decay (NMD) is an important element in alternative splicing regulation (29) and is associated with self-regulatory mechanisms: 
- $\quad$ Polypyrimidine tract binding protein (PTB) regulates its own expression through a negative-feedback loop involving alternative splicing, which requires binding to mRNA and subsequent NMD triggered by exon skipping (30). PTB autogenous interaction is particularly relevant because over-expression of the protein results in cell toxicity $(31,32)$.

- Similarly, heterogeneous nuclear ribonucleoprotein L hnRNP-L is able to induce NMD by associating with its mRNA (33). Our predictions, carried out with catRAPID fragments ("Long RNA" fragmentation option) indicate that hnRNP-L interacts with its own transcript in three different intronic regions located between exons 1-2, 6-7 and 9-10, which is in complete agreement with experimental evidence (33). More specifically, we predict that hnRNP-L protein binds with high affinity to the 3 ' CA cluster $6 \mathrm{~A}$ of the hnRNPL gene (intron 6) and not to sequence $6 \mathrm{~A}$ (negative control), which is perfectly in agreement with the results of in vitro splicing assays performed by Rossbach et al. (33).

These and other results indicate that autogenous interactions occur in UTR/intronic regions and play a role in controlling protein production (17).

\subsection{Methods}

The catRAPID omics algorithm performs high-throughput predictions of protein-RNA interactions. catRAPID omics enables: (1) the calculation of protein-RNA interactions on a large scale (up to 105 associations) in a reasonable time; (2) the submission of protein and RNA sequences without any length restriction and (3) to focus on specific protein regions are able to bind to nucleic acid molecules (34),(4) (Table 2; see Notes 1.0 and 2.0). catRAPID omics is based on the catRAPID algorithm, which exploits the contributions of secondary structure, hydrogen bonding and van der Waals' interactions to predict protein-RNA associations (12).

- The time required by the original catRAPID algorithm for predicting a single RNA-protein interaction strictly depends on the features of the input molecules, which are computed on the 
fly for each submission (using parallel calculation, $<10$ minutes are required for proteomic interactions of one RNA molecule).

- To speed up the calculation of a far greater number of interactions, we introduce in catRAPID omics a system of organism-specific feature libraries.

\subsection{Notes}

catRAPID is interfaced with other methods to improve its predictive power (35). Very recent implementations include analysis of co-expression networks (36) the cleverSuite approach to predict the RNA-binding ability of proteins (37) and the SeAMoTe algorithm to identify regulatory elements coding / non-coding transcripts (38) :

1.0 To train the cleverSuite (http://s.tartaglialab.com/page/clever_suite), we focused on RNAinteracting proteins detected with UV $\mathrm{CCL}$ and PAR-CL protocols on proliferating HeLa cells followed by sequencing and compared them with the rest of cell lysate (39). Analysis of physico-chemical properties revealed a strong and consistent RNA binding property of the dataset (RNA-binding scales (39-41) discriminate $32-35 \%$ of the entire database). The cleverSuite selects the scales for nucleic acid binding (40,41), membrane (42), burial (43) and aggregation (44) propensities, achieving a sensitivity of 0.72 and false positive rate of 0.24 on the entire dataset. We applied the cleverSuite to proteins that are classified as putative RNA-binding because they lack the canonical RNA-binding domains (39). We observed correct classification associated with a sensitivity of 0.83 and false positive rate of 0.15 , which indicates very high agreement with experimental data.

2.0 Detection of regulatory motifs is a challenging task. For this reason, we developed the SeAMotE algorithm (http://s.tartaglialab.com/new_submission/seamote) (38), which provides an easy-to-use interface and allows the exhaustive analysis of large-scale datasets. Our approach offers unique features such as the discrimination based on the actual occurrences (i.e. pattern counts are not estimated) in the datasets, the choice of multiple reference backgrounds (shuffle, random or custom) and the output of the most significant motifs in the 
whole span of tested motif widths, thus providing a wide range of solutions. In conclusion, our web-server is a powerful tool for the identification of enriched sequence patterns that characterize recognition process between proteins and nucleic acids. To evaluate SeAMotE performances on large-scale datasets, we collected recent CLIP experiments and assessed ability to identify significantly enriched motifs (Fisher's exact test). In each case analyzed, we compared RNAs bound to a specific protein (foreground set) with the same amount of noninteracting transcripts (background set). The DREME (45) algorithm was used as a reference to evaluate the performance of our system. Our method achieves both higher discrimination, which is the ability to separate the foreground from the background set, and significance, denoted by lower P-values associated with sequence motifs. In addition, SeAMoTe also shows very high sensitivity ( 90\%) and accuracy (80\%). 
Table 1 - Algorithms of the catRAPID suite. Computational models, their applications as well as example are showed here.

Table 2 - Composition of reference libraries used in catRAPID omics. Proteomes are divided in full-length (protein between 50 and 750 amino acids in length) and domains (derived from proteins $>50$ amino acids in length). Both sets are divided in additional groups, based on the ability of the entries to bind to RNA or DNA. Transcriptome searches use RNA categories coding and noncoding, depending on the entries annotation on ENSEMBL version 68. Length of the transcripts in the datasets ranges from 50 to 1200 nucleotides, but larger RNA can be added to libraries.

Figure 1 - UNR autogenous interactions. UNR transcript and its 5' UTR (nucleotides 1-261) bind to UNR protein (27). A) Our calculations, carried out with catRAPID fragments ("Protein and RNA uniform fragmentation" option), recapitulate experimental results in great detail, identifying cold shock domains (CSD; Figure 1a) (28) involved in autogenous interaction; B) In agreement with experimental evidence (27), UNR protein is predicted to bind to purine repeats, such as the guanine-rich region of UNR 5 ' UTR. 
1. Ascano M, Gerstberger S, Tuschl T (2013) Multi-disciplinary methods to define RNA-protein interactions and regulatory networks. Curr Opin Genet Dev 23(1):20-28.

2. Lunde BM, Moore C, Varani G (2007) RNA-binding proteins: modular design for efficient function. Nat Rev Mol Cell Biol 8(6):479-490.

3. Baltz AG, et al. (2012) The mRNA-Bound Proteome and Its Global Occupancy Profile on Protein-Coding Transcripts. Molecular Cell 46(5):674-690.

4. Castello A, et al. (2012) Insights into RNA biology from an atlas of mammalian mRNA-binding proteins. Cell 149(6):1393-1406.

5. Kwon SC, et al. (2013) The RNA-binding protein repertoire of embryonic stem cells. Nat Struct Mol Biol 20(9):1122-1130.

6. Terribilini M, et al. (2007) RNABindR: a server for analyzing and predicting RNA-binding sites in proteins. Nucleic Acids Res 35(Web Server issue):W578-584.

7. Fernandez M, et al. (2011) Prediction of dinucleotide-specific RNA-binding sites in proteins. BMC Bioinformatics 12 Suppl 13:S5.

8. Bellucci M, Agostini F, Masin M, Tartaglia GG (2011) Predicting protein associations with long noncoding RNAs. Nat Methods 8(6):444-445.

9. Hofacker IL (2003) Vienna RNA secondary structure server. Nucleic Acids Research 31(13):3429 -3431.

10. Lorenz R, et al. (2011) ViennaRNA Package 2.0. Algorithms Mol Biol 6:26.

11. Doshi KJ, Cannone JJ, Cobaugh CW, Gutell RR (2004) Evaluation of the suitability of freeenergy minimization using nearest-neighbor energy parameters for RNA secondary structure prediction. BMC Bioinformatics 5:105.

12. Bellucci M, Agostini F, Masin M, Tartaglia GG (2011) Predicting protein associations with long noncoding RNAs. Nat Methods 8(6):444-445.

13. Cirillo D, Agostini F, Tartaglia GG (2013) Predictions of protein-RNA interactions. Wiley Interdisciplinary Reviews: Computational Molecular Science 3(2):161-175.

14. Agostini F, Cirillo D, Bolognesi B, Tartaglia GG (2013) X-inactivation: quantitative predictions of protein interactions in the Xist network. Nucleic Acids Res 41(1):e31.

15. Cirillo D, et al. (2013) Neurodegenerative diseases: Quantitative predictions of protein-RNA interactions. RNA 19(2):129-140.

16. Johnson R, Noble W, Tartaglia GG, Buckley NJ (2012) Neurodegeneration as an RNA disorder. Prog Neurobiol 99(3):293-315.

17. Zanzoni A, et al. (2013) Principles of self-organization in biological pathways: a hypothesis on the autogenous association of alpha-synuclein. Nucl Acids Res:gkt794.

18. Agostini F, et al. (2013) catRAPID omics: a web server for large-scale prediction of proteinRNA interactions. Bioinformatics:btt495.

19. Ayala YM, et al. (2011) TDP-43 regulates its mRNA levels through a negative feedback loop. EMBO J 30(2):277-288. 
20. Schaeffer C, et al. (2001) The fragile $X$ mental retardation protein binds specifically to its mRNA via a purine quartet motif. EMBO J 20(17):4803-4813.

21. Tartaglia GG, Pechmann S, Dobson CM, Vendruscolo M (2007) Life on the edge: a link between gene expression levels and aggregation rates of human proteins. Trends Biochem Sci 32(5):204-6.

22. Baldwin AJ, et al. (2011) Metastability of native proteins and the phenomenon of amyloid formation. J Am Chem Soc 133(36):14160-14163.

23. Ewen ME, Miller SJ (1996) p53 and translational control. Biochim Biophys Acta 1242(3):181184.

24. Xu J, et al. (2011) Gain of function of mutant p53 by coaggregation with multiple tumor suppressors. Nat Chem Biol 7(5):285-295.

25. Balakrishnan K, De Maio A (2006) Heat shock protein 70 binds its own messenger ribonucleic acid as part of a gene expression self-limiting mechanism. Cell Stress Chaperones 11(1):44-50.

26. Parakhnevitch NM, Ivanov AV, Malygin AA, Karpova GG (2007) Human ribosomal protein S13 inhibits splicing of its own pre-mRNA. Mol Biol 41(1):44-51.

27. Schepens B, et al. (2007) A role for hnRNP C1/C2 and Unr in internal initiation of translation during mitosis. EMBO J 26(1):158-169.

28. Triqueneaux G, Velten M, Franzon P, Dautry F, Jacquemin-Sablon H (1999) RNA binding specificity of Unr, a protein with five cold shock domains. Nucleic Acids Res 27(8):19261934.

29. Kalyna M, et al. (2011) Alternative splicing and nonsense-mediated decay modulate expression of important regulatory genes in Arabidopsis. Nucl Acids Res:gkr932.

30. Wollerton MC, Gooding C, Wagner EJ, Garcia-Blanco MA, Smith CWJ (2004) Autoregulation of polypyrimidine tract binding protein by alternative splicing leading to nonsense-mediated decay. Mol Cell 13(1):91-100.

31. Lin S, Wang MJ, Tseng K-Y (2013) Polypyrimidine tract-binding protein induces p19(Ink4d) expression and inhibits the proliferation of H1299 cells. PLOS ONE 8(3):e58227.

32. Izquierdo JM, et al. (2005) Regulation of Fas alternative splicing by antagonistic effects of TIA-1 and PTB on exon definition. Mol Cell 19(4):475-484.

33. Rossbach O, et al. (2009) Auto- and cross-regulation of the hnRNP L proteins by alternative splicing. Mol Cell Biol 29(6):1442-1451.

34. Finn RD, et al. (2009) The Pfam protein families database. Nucleic Acids Research 38(Database):D211-D222.

35. Cirillo D, Livi CM, Agostini F, Tartaglia GG (2014) Discovery of protein-RNA networks. Mol Biosyst 10(7):1632-1642.

36. Cirillo D, et al. (2014) Constitutive patterns of gene expression regulated by RNA-binding proteins. Genome Biol 15(1):R13.

37. Klus $P$, et al. (2014) The cleverSuite approach for protein characterization: predictions of structural properties, solubility, chaperone requirements and RNA-binding abilities. Bioinformatics 30(11):1601-1608.

38. Agostini F, Cirillo D, Ponti RD, Tartaglia GG (2014) SeAMotE: a method for high-throughput motif discovery in nucleic acid sequences. BMC Genomics 15(1):925. 
39. Castello A, et al. (2012) Insights into RNA Biology from an Atlas of Mammalian mRNA-Binding Proteins. Cell 149(6):1393-1406.

40. Terribilini M, et al. (2006) Prediction of RNA binding sites in proteins from amino acid sequence. RNA 12(8):1450-1462.

41. Lewis BA, et al. (2011) PRIDB: a protein-RNA interface database. Nucleic Acids Res 39(Database issue):D277-D282.

42. Nakashima H, Nishikawa K, Ooi T (1990) Distinct character in hydrophobicity of amino acid compositions of mitochondrial proteins. Proteins 8(2):173-178.

43. Wertz DH, Scheraga HA (1978) Influence of water on protein structure. An analysis of the preferences of amino acid residues for the inside or outside and for specific conformations in a protein molecule. Macromolecules 11(1):9-15.

44. Pawar AP, et al. (2005) Prediction of "aggregation-prone" and "aggregation-susceptible" regions in proteins associated with neurodegenerative diseases. J Mol Biol 350(2):379-92.

45. Bailey TL (2011) DREME: motif discovery in transcription factor ChIP-seq data. Bioinformatics 27(12):1653-1659. 